\title{
Identification of the dissipated energy by friction in dynamic multibody systems
}

\author{
Z.-Q. Feng ${ }^{1, a}$, J.-M. Cros $^{1}$, C. Renaud ${ }^{1}$ and G. Turbelin ${ }^{1}$ \\ Université d'Evry-Val d'Essonne, Laboratoire de Mécanique et d'Energétique d'Evry, 40 rue du Pelvoux, 91020 Evry, France
}

Received 30 October 2007, Accepted 15 February 2008

\begin{abstract}
This paper is devoted to the modeling of finite deformations of elastic bodies under contact/impact conditions. In this kind of applications, it is well known that the total energy will be reduced versus time because of frictional effects. However, how to identify the dissipated energy in a quantitative way remains an open question. The aim of this work is to propose a numerical solution to this question. The proposed Bi-First algorithm combines the bi-potential method for contact modeling in dynamic cases and the first order algorithm for integration of the equation of motion. Numerical examples involve the impact of an elastic plate onto a rigid surface and the impact between two hyperelastic blocks and a rigid wall. The dissipated energy versus time will be plotted and the influence of friction coefficient on the dissipated energy will be discussed.
\end{abstract}

Key words: Contact/Impact; energy dissipation; numerical modeling.

\section{Introduction}

Problems involving contact and friction are among the most difficult ones in mechanics and at the same time of crucial practical importance in many engineering branches. A large number of algorithms for the modeling of contact problems by the finite element method have been presented in the literature. See for example the monographs by Kikuchi and Oden [9], Zhong [19], Wriggers [17], Laursen [13] and the references therein. De Saxcé and Feng [1] have proposed a bi-potential method, in which an augmented Lagrangian formulation was developed. Feng et al. [3,5] have successfully applied this method for the modeling of static contact problems between elastic and Blatz-Ko hyperelastic bodies.

For dynamic implicit analysis in structural mechanics, the most commonly used time integration algorithm is the second order algorithm such as Newmark, Wilson, HHT. Wriggers et al. [18] have developed a radial return mapping scheme to deal with impact-contact problems. Laursen et al. $[12,14,15]$ have considered dynamic impact under the auspices of a conservative system and have proposed the means to address the dynamic contact conditions so that they preserve the global conservation properties. The integration scheme is based on the second order algorithm. The first order algorithm has also been proposed by Jean [7] for time stepping in rigid-body dynamic contact problems. Recently, Feng et al. [6] have applied

\footnotetext{
${ }^{a}$ Corresponding author: feng@iup.univ-evry.fr
}

this algorithm for the modeling of impact problems between elastic bodies. The aim of the present paper is to apply the so-called Bi-First algorithm for contact modeling in dynamic cases between elastic and hyperelastic materials. In this kind of applications, it is well known that the total energy will be reduced versus time because of frictional effects. However, how to identify the dissipated energy in a quantitative way remains an open question. So our work is to propose a numerical approach to answer this question. Two numerical examples are performed in this study to show the validity and efficiency of the algorithm developed. The first example concerns the oblique impact of a 2D elastic plate onto a rigid surface with rebounding. The second example simulates the impact between hyperelastic and rigid bodies. Both frictionless and frictional contacts are considered to highlight the physical energy dissipation by frictional effects.

\section{Local contact modeling}

For notational convenience, we assume that the contact with friction may occur between some points of two bodies $\mathrm{A}$ and $\mathrm{B}$. The contact and friction laws are written in terms of relative velocity $\dot{\mathbf{u}}=\dot{\mathbf{u}}_{A}-\dot{\mathbf{u}}_{B}$ and of contact reactions $\mathbf{r}$. The following contact bi-potential is introduced by De Saxcé and Feng [1]:

$$
b_{c}(-\dot{\mathbf{u}}, \mathbf{r})=\bigcup_{\mathbb{R}_{-}}\left(-\dot{u}_{n}\right)+\bigcup_{K_{\mu}}(\mathbf{r})+\mu r_{n}\left\|-\dot{\mathbf{u}}_{t}\right\|
$$


where $\left.\left.\mathbb{R}_{-}=\right]-\infty, 0\right], K_{\mu}$ is the Coulomb's cone and $\bigcup$ stands for the indicator function. In order to avoid nondifferentiable potentials that occur in contact problems, it is convenient to use the Augmented Lagrangian Method $[1,16]$. For the contact bi-potential $b_{c}$, we have:

$$
\forall \mathbf{r}^{\prime} \in K_{\mu}, \quad \varrho \mu\left(r_{n}^{\prime}-r_{n}\right)\left\|\dot{\mathbf{u}}_{t}\right\|+\left(\mathbf{r}^{\prime}-(\mathbf{r}-\varrho \dot{\mathbf{u}})\right) \cdot\left(\mathbf{r}^{\prime}-\mathbf{r}\right) \geq 0
$$

where $\varrho$ is a solution parameter which is not user-defined. The inequality (2) means that $\mathbf{r}$ is the projection of $\boldsymbol{\tau}$ onto the closed convex Coulomb's cone:

$$
\mathbf{r}=\operatorname{proj}\left(\tau, K_{\mu}\right)
$$

For the numerical solution of the implicit equation (3), Uzawa's algorithm can be used, which leads to an iterative process involving one predictor-corrector step:

$$
\begin{aligned}
& \text { Predictor } \boldsymbol{\tau}^{i+1}=\mathbf{r}^{i}-\varrho^{i}\left(\dot{\mathbf{u}}_{t}^{i}+\left(\dot{u}_{n}^{i}+\mu\left\|\dot{\mathbf{u}}_{t}^{i}\right\|\right) \mathbf{n}\right) \\
& \text { Corrector } \mathbf{r}^{i+1}=\operatorname{proj}\left(\boldsymbol{\tau}^{i+1}, K_{\mu}\right)
\end{aligned}
$$

More recently, a Newton type method is also proposed to solve the system of non linear equations [8].

\section{Global time stepping}

Generally, mechanical behaviors of solids under contact/impact conditions are governed by a set of nonlinear equations

$$
\mathbf{M} \ddot{\mathbf{u}}=\mathbf{F}+\mathbf{R}, \quad \text { where } \mathbf{F}=\mathbf{F}_{\text {ext }}-\mathbf{F}_{\text {int }}
$$

where $\mathbf{M}$ is the mass matrix, $\mathbf{F}_{\text {ext }}$ the applied forces vector, $\mathbf{F}_{\text {int }}$ the internal forces vector and $\mathbf{R}$ the reaction forces vector. Taking the derivative of $\mathbf{F}_{\text {int }}$ with respect to the nodal displacements $\mathbf{u}$ gives the tangent stiffness matrix $\mathbf{K}$. The most common method to integrate equation (5) is the Newmark method which is based on a second order algorithm. However, in impact problems, higher order approximation does not necessarily mean better accuracy, and may even be superfluous. At the moment of a sudden change of contact conditions (impact, release of contact), the velocity and acceleration are not continuous, and excessive regularity constraints may lead to serious errors. For this reason, Jean [7] has proposed a first order algorithm which is used in this work. This algorithm is based on the following approximations:

$$
\begin{gathered}
\int_{t}^{t+\Delta t} \mathbf{M} d \dot{\mathbf{u}}=\mathbf{M}\left(\dot{\mathbf{u}}^{t+\Delta t}-\dot{\mathbf{u}}^{t}\right) \\
\int_{t}^{t+\Delta t} \mathbf{F} d t=\Delta t\left((1-\xi) \mathbf{F}^{t}+\xi \mathbf{F}^{t+\Delta t}\right) \\
\int_{t}^{t+\Delta t} \mathbf{R} d t=\Delta t \mathbf{R}^{t+\Delta t} \\
\mathbf{u}^{t+\Delta t}-\mathbf{u}^{t}=\Delta t\left((1-\theta) \dot{\mathbf{u}}^{t}+\theta \dot{\mathbf{u}}^{t+\Delta t}\right)
\end{gathered}
$$

where $0 \leq \xi \leq 1 ; 0 \leq \theta \leq 1$. In the iterative solution procedure, all the values at time $t+\Delta t$ are replaced by the values of the current iteration $i+1$. Without going into details, we obtain the recursive form of (5) in terms of displacements:

$$
\begin{aligned}
\overline{\mathbf{K}}^{i} \Delta \mathbf{u} & =\overline{\mathbf{F}}^{i}+\overline{\mathbf{F}}_{a c c}^{i}+\mathbf{R}^{i+1} \\
\mathbf{u}^{i+1} & =\mathbf{u}^{i}+\Delta \mathbf{u}
\end{aligned}
$$

where the so-called effective terms are given by

$$
\begin{gathered}
\overline{\mathbf{K}}^{i}=\xi \mathbf{K}^{i}+\frac{1}{\theta \Delta t^{2}} \mathbf{M}^{i} \\
\overline{\mathbf{F}}_{a c c}^{i}=-\frac{1}{\theta \Delta t^{2}} \mathbf{M}^{i}\left\{\mathbf{u}^{i}-\mathbf{u}^{t}-\Delta t \dot{\mathbf{u}}^{t}\right\} \\
\overline{\mathbf{F}}^{i}=(1-\xi)\left(\mathbf{F}_{\text {int }}^{t}+\mathbf{F}_{\text {ext }}^{t}\right)+\xi\left(\mathbf{F}_{\text {int }}^{i}+\mathbf{F}_{\text {ext }}^{t+\Delta t}\right) .
\end{gathered}
$$

\section{Energy computation}

After determining the displacement and the velocity fields, we can calculate different energies. First, we calculate the strain energy density $W$ on each integration point (Gauss point) of a finite element. The total elastic strain energy of the contact bodies (discretized by $n_{e l}$ finite elements) is then written by

$$
E_{e}=\sum_{e=1}^{n_{e l}} \int_{V_{e}} W d V .
$$

The total kinetic energy can be calculated at the global level by

$$
E_{k}=\frac{1}{2} \dot{\mathbf{u}}^{T} \mathbf{M} \dot{\mathbf{u}} .
$$

Finally, the total energy of the system of solids is

$$
E_{t}=E_{e}+E_{k}
$$

The case of interest for the analysis presented below corresponds to the homogeneous Neumann problem, characterized by no imposed boundary displacements and no external loading. In addition, if frictionless contact is considered, the total energy should be conserved. The given example shows that this fundamental energy conservation property is preserved.

In case of frictional impact problems, the total energy will decrease due to friction. For a given time interval $[0, T]$, the dissipated energy is explicitly calculated by

$$
\Delta E_{t}=E_{t_{T}}-E_{t_{0}} .
$$

\section{Numerical results}

The Bi-First algorithm presented above has been implemented and tested in the finite element code FER/Impact $[2,4]$. We present here two examples of application. 


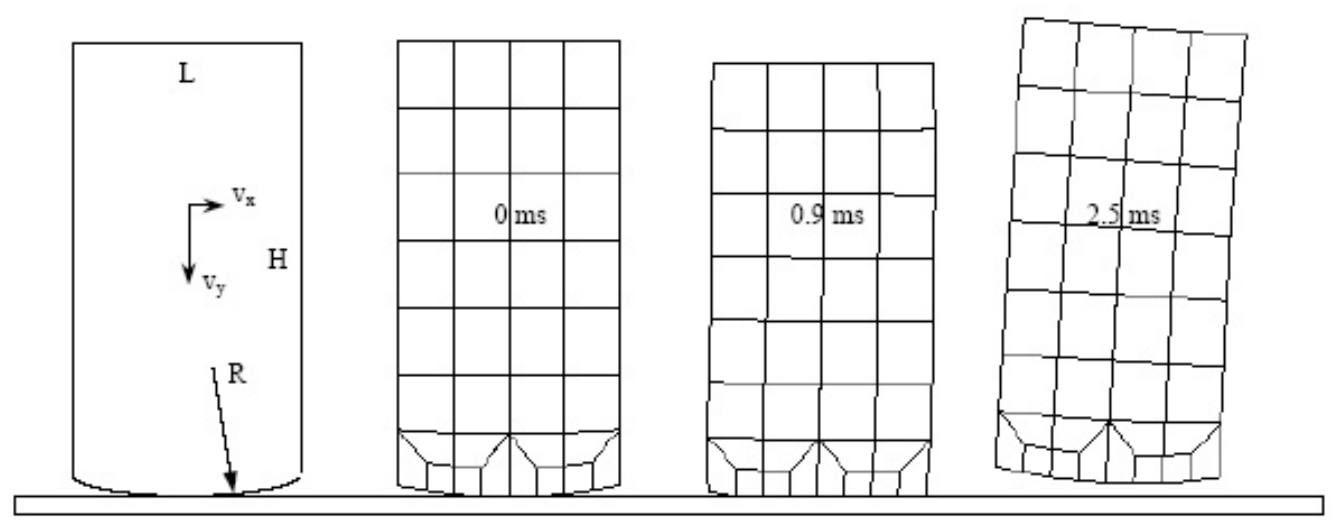

Fig. 1. Oblique impact of an elastic plate: geometry and deformed shapes vs. time.

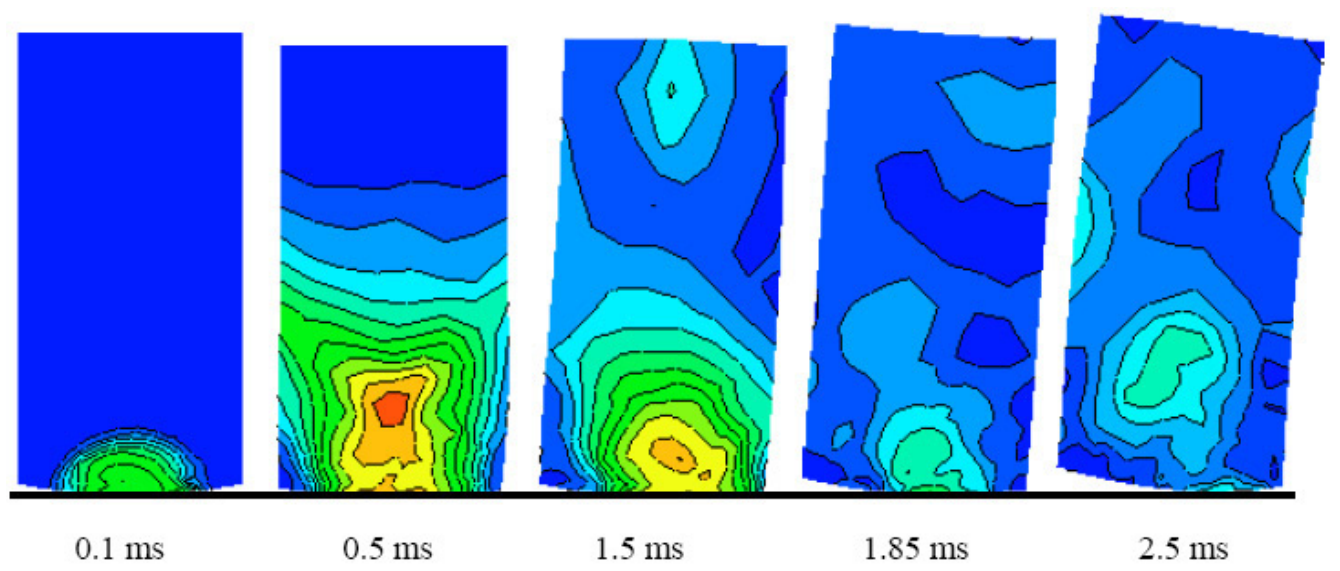

Fig. 2. Evolution of von Mises stress versus time.

\subsection{Oblique impact of an elastic plate with rebounding}

The first example of dynamics analysis will be presented to show the validity and efficiency of the model developed. The problem concerns the oblique impact of an elastic plate onto a rigid surface with rebounding. This example has been proposed and studied by Kwak et al. [10,11] using Linear Complementarity Problem (LCP) formulation. The geometric configuration and successive deformed meshes are displayed in Figure 1. The characteristics of this example are: Young's modulus $E=10^{7} \mathrm{~Pa}$, Poisson's ratio $v=0.25$, mass density $\rho=1000 \mathrm{~kg} / \mathrm{m}^{3}$, Friction coefficient $\mu=0.1$, Initial velocity: $v_{x}=3 \mathrm{~m} / \mathrm{s}$, $v_{y}=-5 \mathrm{~m} / \mathrm{s}$. The geometric sizes are: $L=0.04 \mathrm{~m}$, $H=0.08 \mathrm{~m}$, radius $R=0.101 \mathrm{~m}$, thickness $e=0.01$ $\mathrm{m}$. The total simulation time is $3 \times 10^{-3} \mathrm{~s}$ and the solution parameters are: $\Delta t=10^{-5} \mathrm{~s}, \xi=\theta=0.5$. The plate is modeled by 54 nodes and 37 linear quadrilateral plane stress elements (Fig. 1). Figure 2 shows the evolution of von Mises stress contours in the plate. The maximum value is $923000 \mathrm{~Pa}$. Figure 3 shows the plots of the kinetic energy $E_{k}$, the elastic deformation energy $E_{e}$ and the total energy $E_{t}=E_{k}+E_{e}$. It is noted from Figure 3 that the total energy is dissipated by frictional effects and the dissipated energy is identified quantitatively. On the

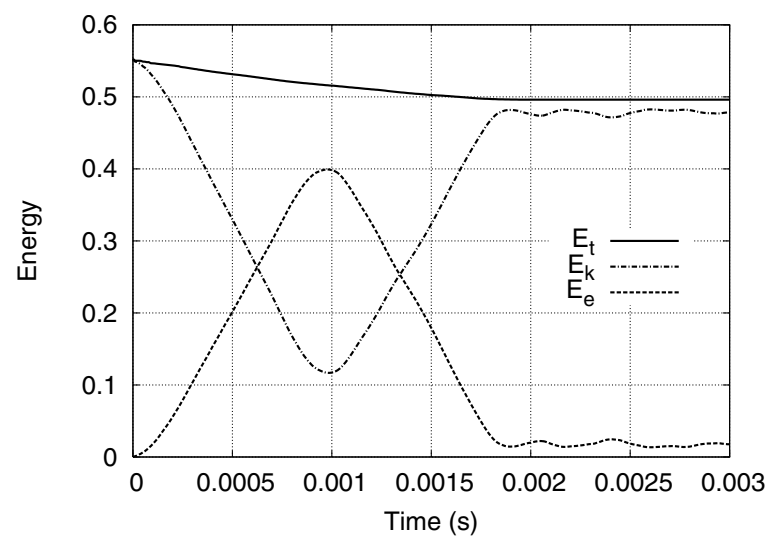

Fig. 3. Energy evolution with friction.

other hand, if frictionless impact is considered, the total energy is quite well conserved as shown in Figure 4. It is also interesting to examine another question: is the dissipated energy monotone to the friction coefficient? The answer is negative according to numerical results. The proof is illustrated by Figure 5 which shows the evolution of the dissipated energy with respect to the friction 


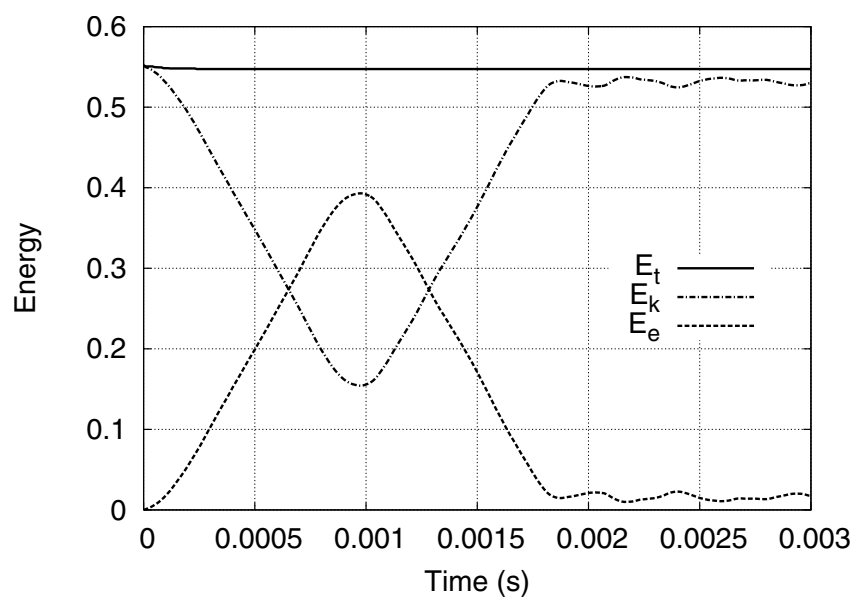

Fig. 4. Energy evolution without friction.

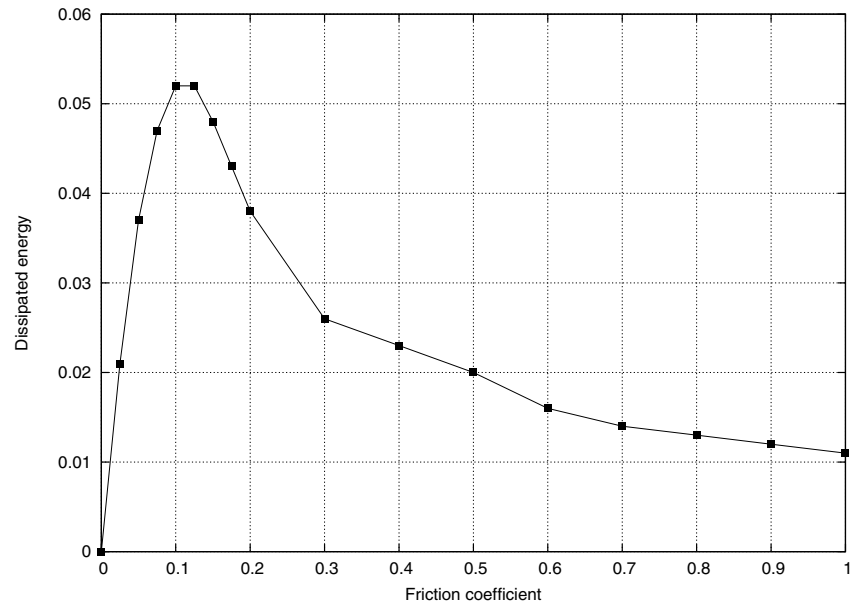

Fig. 5. Evolution of the dissipated energy with respect to the friction coefficient.

coefficient. In fact, when the friction coefficient increases, the friction forces increase. However, the tangential slips will decrease. We know that the dissipated energy depends not only on the friction forces but also on the tangential slips on the contact nodes, so it is understandable to have the behavior as shown in Figure 5 .

\subsection{Impact between two hyperelastic bodies and a rigid wall}

The second example simulates the impact with friction and large strains between hyperelastic bodies of Blatz-Ko model. In doing so, we wish to further explore the performance of the present method in a general situation with more complicated contact sequences. Three contact bodies are involved in this problem as shown in Figure 6. The cylinder is given an initial velocity of $v_{x}=-10 \mathrm{~m} / \mathrm{s}$; $v_{y}=-15 \mathrm{~m} / \mathrm{s}$, hitting the block and afterwards the rigid wall which is freely posed on the foundation. The cylinder has an inner diameter of $1.5 \mathrm{~cm}$ and an outer diameter of $2.5 \mathrm{~cm}$. The block has a dimension of $16 \times 6 \mathrm{~cm}$.

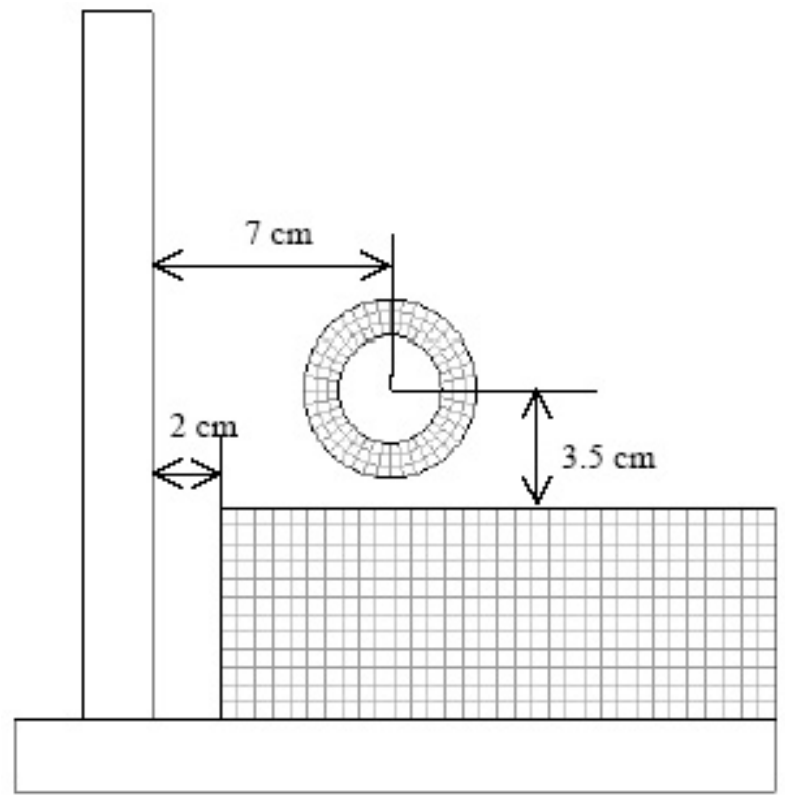

Fig. 6. Initial configurations and meshes.

The rigid wall has a dimension of $2 \times 20 \mathrm{~cm}$. The material constants are: shear modulus $G=1 \mathrm{MPa}$, mass density $\rho=1000 \mathrm{~kg} / \mathrm{m}^{3}$ (cylinder); $G=2 \mathrm{MPa}, \rho=$ $1200 \mathrm{~kg} / \mathrm{m}^{3}$ (block); $\rho=2700 \mathrm{~kg} / \mathrm{m}^{3}$ (wall). The coefficient of Coulomb friction is 0.4. The total simulation time is $0.02 \mathrm{~s}$ and the solution parameters are: $\Delta t=10^{-4} \mathrm{~s}$, $\xi=\theta=0.5$. Figure 7 shows the deformed configurations of the system at different times. Figure 8 shows the plots of the kinetic energy $E_{k}$, the elastic strain energy $E_{e}$ and the total energy $E_{t}$. We can observe clearly that the total energy is dissipated during the impact process due to frictional effects. After the release of contact, the total energy tends to a constant value as expected, although it exists oscillations of the kinetic energy and of the elastic strain energy. In fact, the oscillations result from the hyperelastic behavior of the materials.

\section{Conclusion}

The main purpose of this paper is to briefly present the recent development of the bi-potential method applied to dynamic analysis of contact problems with Coulomb friction between hyperelastic and rigid bodies. The Bi-First algorithm for local analysis of frictional contact problems and for global time integration of dynamics equations is suitable for a wide range of engineering applications. From numerical experiments on two impact problems, we have observed that the total energy is well conserved for frictionless impact and the algorithm allows to identify quantitatively the physical energy dissipation by friction. In addition, we have shown that the dissipated energy is not a monotonic function of the friction coefficient. 


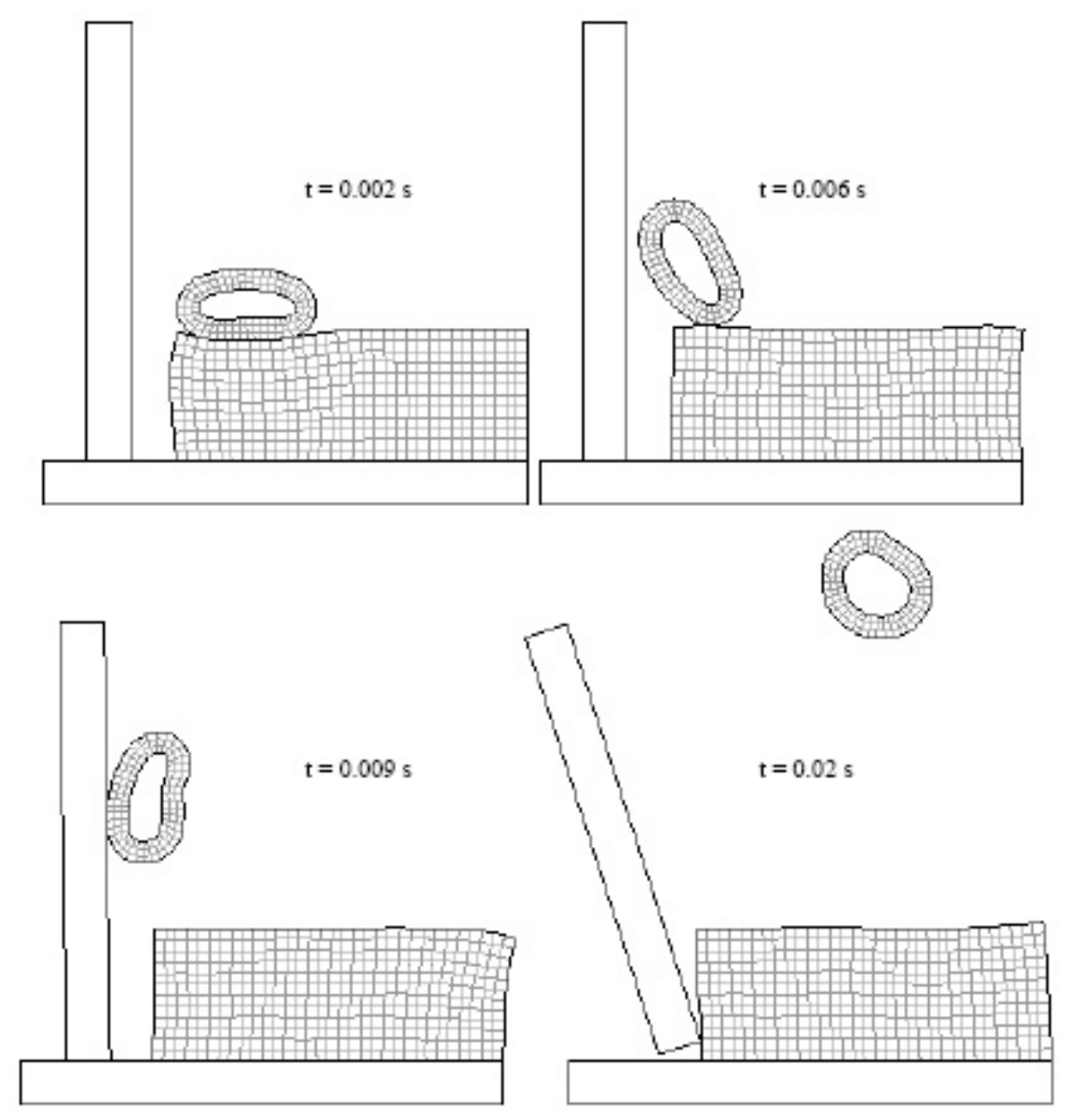

Fig. 7. Deformed configurations at different times.

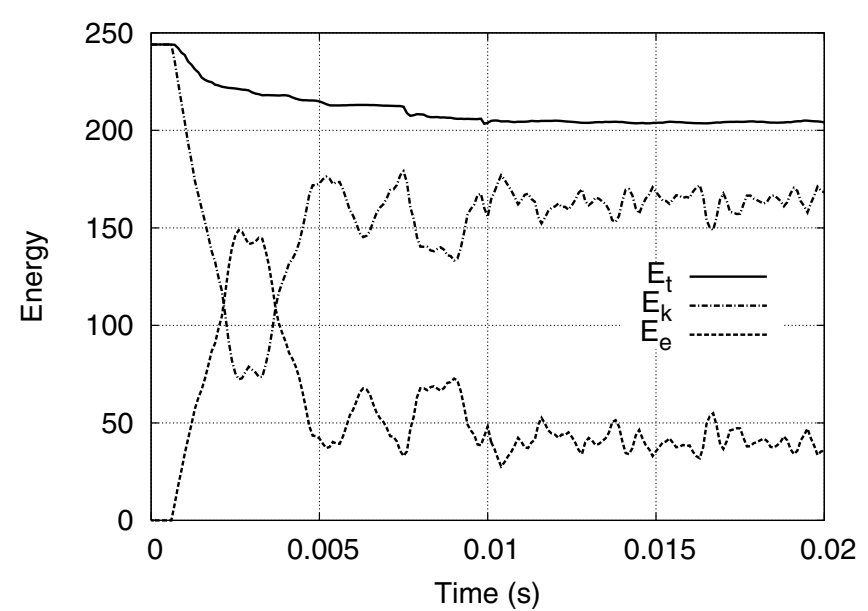

Fig. 8. Energy evolution vs. time.

\section{References}

1. G. de Saxcé, Z.Q. Feng, Math. Comput. Model. 28, 225 (1998)

2. Z.Q. Feng, http://gmfe16.cemif.univ-evry.fr:8080/ feng/ FerImpact.html

3. Z.Q. Feng, Math. Comput. Model. 28, 469 (1998)

4. Z.Q. Feng, B. Magnain, J.M. Cros, Revue Européenne de Mécanique Numérique 15, 175 (2006)
5. Z.Q. Feng, F. Peyraut, N. Labed, Int. J. Eng. Sci. 41, 2213 (2003)

6. Z.Q. Feng et al., Comput. Mech. 36, 375 (2005)

7. M. Jean, Comp. Meth. Appl. Mech. Eng. 177, 235 (1999)

8. P. Joli, Z.Q. Feng, Int. J. Numer. Meth. Eng. 73, 317 (2008)

9. N. Kikuchi, J.T. Oden, A study of variational inequalities and finite elements (Philadelphia: SIAM, 1988).

10. J.O. Kim, B. Kwak, Int. J. Solids, Struct. 33, 4605 (1996)

11. S.H. Ko, B. Kwak, Comput. Struct. 42, 797 (1992)

12. T. Laursen, V. Chawla, Int. J. Numer. Meth. Eng. 40, 863 (1997)

13. T.A. Laursen, Computational contact and impact mechanics: Fundamentals of Modeling Interfacial Phenomena in Nonlinear Finite Element Analysis (Springer Verlag, 2002)

14. T.A. Laursen, G.R. Love, Int. J. Numer. Meth. Eng. 53, $245(2002)$

15. G.R. Love, T.A. Laursen, Comp. Meth. Appl. Mech. Eng. 192, $2223(2003)$

16. J.C. Simo, T.A. Laursen, Comput. Struct. 42, 97 (1992)

17. P. Wriggers, Computational contact mechanics, (John Wiley \& Sons, 2002).

18. P. Wriggers, T.V. Van, E. Stein, Comput. Struct. 37, 319 (1990)

19. Z.H. Zhong, Finite element procedures in contact-impact problems (Oxford University Press, 1993) 